\title{
Human Kinetoplastid Protozoan Infections: Where Are We Going Next?
}

\begin{abstract}
Alessandra Almeida Filardy ${ }^{1}$, Kamila Guimarães-Pinto ${ }^{1}$, Marise Pinheiro Nunes ${ }^{2}$, Ketiuce Zukeram ${ }^{1}$, Lara Fliess ${ }^{1}$, Ludimila Pereira ${ }^{1}$, Danielle Oliveira Nascimento', Luciana Conde ${ }^{1}$ and Alexandre Morrot ${ }^{2,3 *}$
\end{abstract}

\begin{abstract}
${ }^{1}$ Department of Immunology, Paulo de Góes Microbiology Institute, Federal University of Rio de Janeiro, Rio de Janeiro, Brazil, ${ }^{2}$ Immunoparasitology Laboratory, Oswaldo Cruz Foundation, Oswaldo Cruz Institute, FIOCRUZ, Rio de Janeiro, Brazil, ${ }^{3}$ Tuberculosis Research Center, Faculty of Medicine, Federal University of Rio de Janeiro, Rio de Janeiro, Brazil
\end{abstract}

Kinetoplastida trypanosomatidae microorganisms are protozoan parasites exhibiting a developmental stage in the gut of insect vectors and tissues of vertebrate hosts. During the vertebrate infective stages, these parasites alter the differential expression of virulence genes, modifying their biological and antigenic properties in order to subvert the host protective immune responses and establish a persistent infection. One of the hallmarks of kinetoplastid parasites is their evasion mechanisms from host immunity, leading to disease chronification. The diseases caused by kinetoplastid parasites are neglected by the global expenditures in research and development, affecting millions of individuals in the low and middle-income countries located mainly in the tropical and subtropical regions. However, investments made by public and private initiatives have over the past decade leveraged important lines of intervention that if well-integrated to health care programs will likely accelerate disease control initiatives. This review summarizes recent advances in public health care principles, including new drug discoveries and their rational use with chemotherapeutic vaccines, and the implementation of control efforts to spatially mapping the kinetoplastid infections through monitoring of infected individuals in epidemic areas. These approaches should bring us the means to track genetic variation of parasites and drug resistance, integrating this knowledge into effective stewardship programs to prevent vector-borne kinetoplastid infections in areas at risk of disease spreading.

Keywords: parasitic infection, kinetoplastid protozoans, Trypanosoma cruzi, Leishmania, Trypanosoma brucei

\section{INTRODUCTION}

Protozoan infections are one of the most devastating causes of human death worldwide. These infections are caused by protozoan parasites, microorganisms originally classified in the Kingdom Protozoa, which comprises a diverse group of unicellular eukaryotes (1). Although the majority of the protozoan exists as free-living microorganisms in different aquatic and humid environments, there are many species living in association with host organisms, causing severe human diseases $(1,2)$. This is the case of Kinetoplastid parasites, a group of flagellated protozoans that parasitize most plant and animal species; and cause human diseases with public health threats and socialeconomic effects (3). 
The kinetoplastids are a monophyletic group related to the euglenids. These microorganisms are distinguished from other protozoan groups mainly by the presence of kinetoplasts, a granule that contains "kDNA," a DNA located in the mitochondria, associated with the base of the flagella (3). Three distinct kinetoplastids cause human disease: Trypanosoma brucei [human African trypanosomiasis (HAT) or sleeping sickness], Trypanosoma cruzi (Chagas disease), and Leishmania spp. (leishmaniasis) (3, 4), which are still recognized as neglected tropical diseases (NTDs) by the World Health Organization. These two genera of parasites are found in the blood and/or tissues of infected humans and are transmitted by arthropod vectors (Figure 1) (5).
Although the advances in the development of drug therapies and vector control agents against kinetoplastid diseases (6), new strategies are required for global elimination of epidemies. The main limiting efforts for this accomplishment obviously relies on the global investments in R\&D for these NTDs when compared to other diseases with higher levels of financial support, such as malaria, tuberculosis, and HIV, known as "big three" (7). The analysis of the global sums of expenditures for each of these diseases, and their correlations with the social impact indexes on public health indicates a correlation that goes far beyond the political question (8). Such correlation is proportional to the severity of each disease (Figure 2), as to the metric of the disability-adjusted

\section{Kinetoplastid infection cycles}

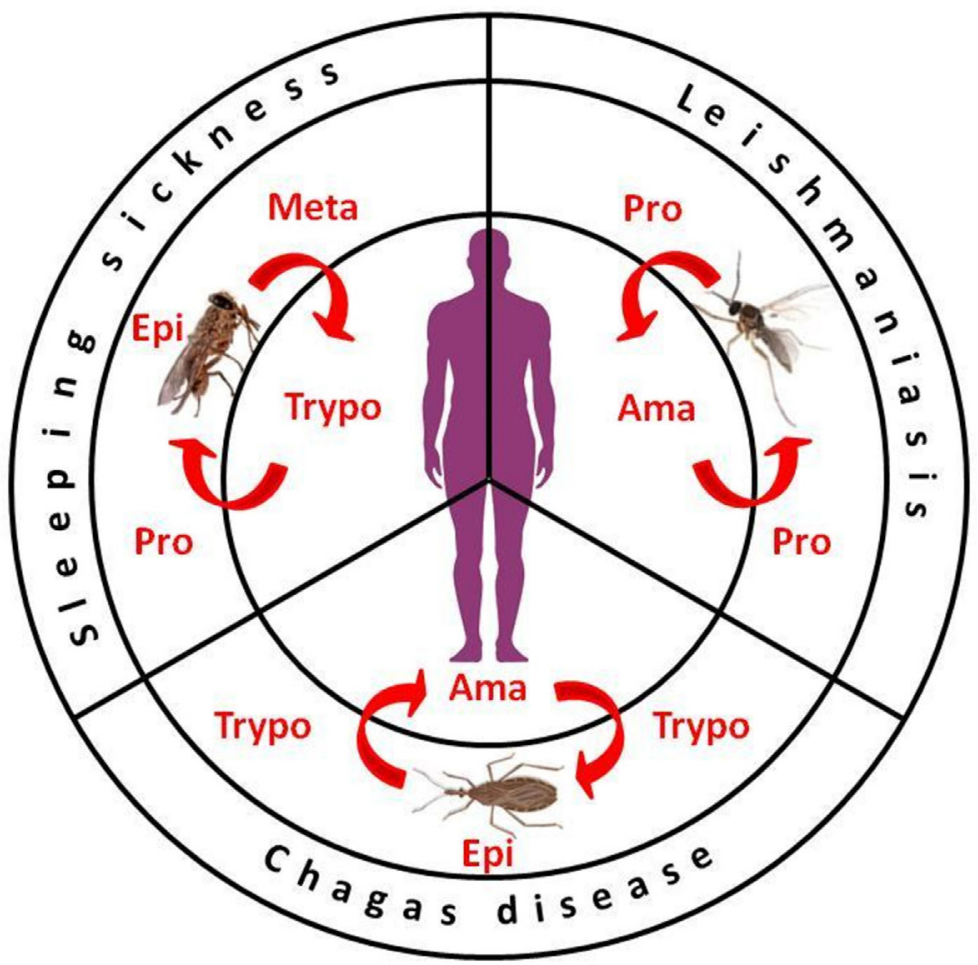

\begin{tabular}{|c|c|c|c|}
\hline Kinetoplastid & Vector & Invertebrate Stages(IS)/Vertebrate stages(VS) & Disease \\
\hline Trypanosoma cruzi & Triatomine bugs & Epimastigotes (IS), metacyclic tripomastigotes (IS/M), trypomastigotes (VS), amastigotes (VS) & Chagas \\
\hline Trypanosama brucei & Tsetse flies & Procyclic trypomastigotes (IS), epimastigotes (IS), metacyclic trypomastigotes (IS/NS), trypomastigotes (NS) & African Trypanosomiasis \\
\hline Leishmania spo & Phlebotomine sandflies & Promastigotes (IV), amastigotes (VS) & Leishmaniasis \\
\hline
\end{tabular}

FIGURE 1 | Kinetoplastid transmission cycles. The life cycles of kinetoplastid protozoan parasites show development stages in both invertebrate and vertebrate hosts. In leishmaniasis, promastigotes (flagellate and mobile forms) are inoculated into the skin along with the saliva of phlebotomine vectors (Diptera: Psychodidae). In the vertebrate hosts, they are maintained inside phagocytic cells under the proliferative form, amastigotes. When ingested by insect vectors, the amastigote forms are transformed into promastigotes which multiply in the intestinal tissue, then migrate as metacyclic promastigotes into the stomodal valve to be later injected into the skin during blood meal. In Chagas' disease, the etiologic agent Trypanosoma cruzi is transmitted to vertebrate hosts as a metacyclic trypomastigote forms by infected triatomine bugs (Triatominae: Reduviidae) during blood feeding. Once in the vertebrate, trypomastigotes differentiate into intracellular amastigote forms. These proliferative stages multiply by binary division, and then differentiate into trypomastigotes, which are released into the bloodstream. When the triatomine bug takes a blood meal from an infected vertebrate host containing circulating parasites, the ingested trypomastigotes forms to differentiate into epimastigotes in the medium intestine of the vector, multiplying by binary division, after which they differentiate into infective metacyclic trypomastigote forms. In sleep sickness or human African trypanosomiasis, the parasite Trypanosoma brucei is transmitted by the bite of the tsetse fly (Glossinidae: Glossina). The parasite exists in the saliva of the invertebrate vector and is injected when the insect feeds on human blood. Unlike Trypanosoma cruzi, trypomastigotes of T. brucei do not invade host cells and, therefore, does not differentiate into intracellular amastigote forms. Instead, T. brucei parasites multiply as trypomastigotes in the blood of infected vertebrate host. The parasite cycle continues when a new vector feeds on a contaminated individual. In the invertebrate vector, the parasites differentiate into proliferative epimastigotes forms, invading the insect salivary glands to continue the cycle. 


\section{Global Funding to Disease Burden}

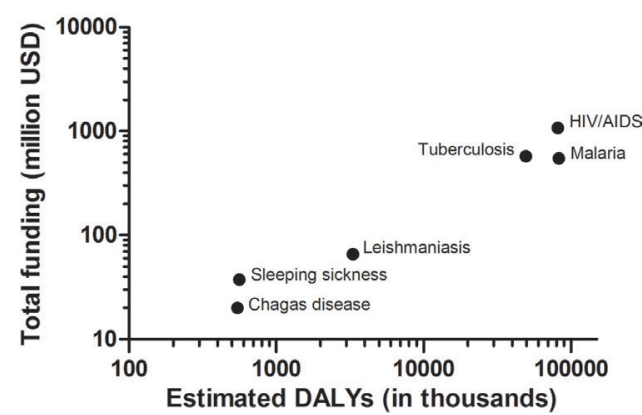

FIGURE 2 | Overall funding for research and development investment correlated with disability-adjusted life years, estimated from the Global Burden of Disease Study.

life year (DALY), an index calculated as the sum of the years of life lost due to premature mortality of patients and the years lost due to disability for people living with the illness (9).

In spite of the low priority of the investments received, studies in the past decades have pointed out the paths for developing effective actions to control human kinetoplastid protozoan infections through a better understanding of the pathogenvertebrate host interactions during their life cycles, disease pathogenesis in the hosts, and methods that allow the diagnosis, even in the acute phase of the infection $(3,4)$. This has provided a better opportunity to prospect new therapeutic targets, more effective drug development approaches, and promising vaccines that, together, may combat these diseases more robustly in the near future (5).

\section{TRENDS IN DRUG DEVELOPMENT FOR KINETOPLASTID INFECTIONS}

Treatment approaches based on drug therapies in kinetoplastid infections are scarce and have been considered to be highly toxic (10). However, interest in research and exploration of new approaches has given a sign of change. Since kinetoplastid infections lead to chronic persistent diseases, it is critical that drug therapy interventions would be focused on the acute phase, with hopes of achieving complete eradication of disease transmission. To that end, it is necessary to establish technical procedures that allow early diagnosis of acute kinetoplastid infection (5). This intervention approach aims to act at a stage of infection in which pathogen has not yet established itself and is present with a low parasitism burden and few down modulatory mechanisms in place over the host immune responses $(1,11,12)$.

In addition, a rapid and efficient intervention during acute phase of the infection, when the host is still under low tissue parasitism, decreases the chances of acquiring drug resistance $(13,14)$. In Chagas disease, benznidazole and nifurtimox have long been the only clinical treatment options for infection (15). However, progress has been made in the research of new promising drugs against the disease. E1224, a pro-drug of ravuconazole, has shown some efficiency in combating the disease (16). The results of the first phase 2 clinical trial in Bolivia, conducted by drugs for neglected diseases initiative, have shown satisfactory protective results for E1224. This drug was effective in controlling T. cruzi parasitism in infected patients. Furthermore, its use in combination with benzanidazole is more effective as compared to monotheraphy-based protocols (16).

To treat HAT, five drugs have been approved: pentamidine, melarsoprol, eflornithine, suramin, and nifurtimox (17). Pentamidine and suramin are used in monotherapies in the early stage of T.b. gambiense and T.b. rhodesiense infections, whereas melarsoprol is used for the second stage of the disease $(18,19)$. Nifurtimox has been used since 2009 in combination with eflornithine, mostly in the second stage of T.b. gambiense infection. The combination therapy protocols for these two drugs have been improved, although there are still practical restrictions to their potential use in large-scale applications (20). Currently, new drugs designed to improve patient care are being considered to meet current elimination targets. Most of them have been optimized to undergo clinical trials (17).

Fexinidazole and oxaborole SCYX-7158, are already being studied in clinical evaluation as oral therapies in phase IIb/III and phase I trials, respectively $(21,22)$. Other important R\&D studies have focused on the virulence responses of the parasite. The cell surface of African trypanosomes (T. brucei spp.) is covered by a dense coat of glycoconjugates that play important roles in the evasion of host immune responses (23). New strategies in drug discovery against HAT aim the development of specific carbohydrate-binding agents capable of inhibiting the action of glycosyltransferases and glycosidases of the parasite, thus altering the nature of the parasite's cell-surface glycans as a treatment target for sleeping sickness (24).

In leishmaniasis, a notable progress in the disease treatment has been made in the preparation of new formulations of amphotericin B, using liposome carriers (25). In addition, new drugs have been extensively studied in clinical trials, although the effects of HIV co-infection in endemic areas contribute to drug unresponsiveness during therapies (26). The use of paromomycin, an aminoglycoside class antibiotic, has also been shown to be efficient at low cost as a first-line drug (27). Alternatively, studies have suggested the effective action of drugs that act on a broad spectrum against Leishmania parasites. This is the case of miltefosine, a drug that is also used in the treatment of dogs with leishmaniasis in Brazil (28). The miltefosine has an inhibitory action on several biological pathways of the parasite, such as cytochrome $\mathrm{C}$ oxidase, synthesis of phosphatidylcholine, and disruption of parasite $\mathrm{Ca}^{2+}$ homeostasis (29-31).

In addition, drug discovery researches for kinetoplastids have benefited from investments made in the field of protozoan genomics. This is clearly seen in the use of data mining, annotation, and analysis of Leishmania parasite genome that has lead to the creation of LeishCyc database (32). This systematic approach will allow a complete mapping of Leishmania transcriptomics, proteomics, and metabolomics enabling the development of new compounds that can be used in high-throughput screening approaches to search selective drugs against Leishmania 
parasites (32). In fact omics-based analyses have facilitated the broadening of $\mathrm{R} \& \mathrm{D}$ researches in the field of drug development. Lipidomics analyses have yielded the characterization of lipid structures, protein lipidation pathways in post-translational modifications, and their putative functions for kinetoplastid parasites $(33,34)$. This knowledge will allow the search for new classes of anti-parasitic pharmaceuticals.

\section{RESEARCH AND DEVELOPMENT OF NEW VACCINES AGAINST KINETOPLASTID PARASITES}

The major challenge in the preventive control of kinetoplastid infections and other neglected tropic infectious diseases is undoubtedly the implementation of low-cost vaccines for public health programs in affected countries, most of them were lowand middle-income countries $(5,35)$. This accomplishment would enable to structure intervention actions in health care at the level of mass treatment programs, thus avoiding disease reemergence. To reach this ideal scenario, there are serious socialpolitical obstacles to overcome in order to ensure the development of clinical tests. The most incisive limitation is unquestionably economic, resulting from a scarce financial incentives and market failures in view of the geopolitical areas where epidemics occur (35). Such constraints are likely to arouse less interest from financial and pharmaceutical institutions in the market development strategies for vaccines against NTDs in general.

There are no licensed vaccines for kinetoplastid infections yet, reinforcing the need for their development, particularly in countries where they are epidemic. Most of the ongoing vaccine studies have been conducted at the level of basic research (5). Particularly, recent advances in the construction of manipulative parasites through genome engineering using CRISPR/Cas9 and Cre recombinase have been dedicated to reprogramming their genome, allowing the identification of regulatory genes associated with the cell fates in the host-parasite interplay (36). These critical virulence genes represent good candidates to be studied as vaccine targets.

Interesting, some kinetoplastid vaccines undergoing clinical trials are promising, since they are cost-effective and show longterm protection against both cutaneous and visceral leishmaniasis. This is the case of vaccines developed by the Infectious Diseases Research Institute, which has included protective Leishmania antigen epitopes in its vaccine formulations used in clinical trials $(37,38)$. Another prominent proposal comes from initiative studies in cooperation with National Institutes of Health. They present a more elaborate vaccine formulation, including protective recombinant Leishmania antigens in combination with sand fly salivary gland antigens, capable of inducing a more robust host immune response, considering vector-host interactions in the transmission of Leishmania parasites (39).

In the case of Chagas disease, initial studies have pointed out potential antigens in experimental mice models capable of inducing host protective immune responses. They were also effective in reducing cardiac parasite loads and disease pathology, increasing host survival indices. Those studies have proposed the use of
Tc24 (calcium binding protein associated with flagellar pocket of T. cruzi) and TSA-1 (Trypomastigote surface antigen-1) by a global consortium (40-42). These are the first candidates to be used in vaccine formulations to prevent Chagas disease. Furthermore, the use of vaccines as a therapeutic approach to treat chagasic patients has also been proposed (42).

\section{KEY CHALLENGES TO KINETOPLASTID PARASITE CONTROL: INTEGRATING NEW APPROACHES INTO ERADICATION STRATEGIES}

To eradicate kinetoplastid infections in humans, we must gain a better understanding of variants of pathogens and their vectors, the transmission models among their hosts, and efficacious preventive vaccine approaches, to understand and design strategies to intervene in the real dynamics of disease spreading in epidemic areas (43). To achieve that, diagnostic tests capable of detecting infections in the acute phase, and accurate analysis of drug resistance in the parasite spreading are essential to trace a better correlation between variants of pathogens and the clinical spectrum of the disease. Another important step of intervention in the transmission cycles of vector diseases is regarding the application of health care models based on geographic information system (GIS) projects and technologies (44). By using this system, we will be able to follow the infection cycle in terms of spatial data, geographical and geodatabases, which allows us to create mapping models to predict potential areas of risk for transmission of vector-borne diseases (45).

The GIS technology is able to compile multi-analysis related to public health data collected from endemic urban and wild areas, including analyses of disease dispersal, genetic variation of parasites, vector and host habitats, and their relationship with microclimates in affected areas. These metadata analyses can be correlated with geographic positioning system to establish interventions of potential infections, thus allowing for a rational use of medications to prevent the emergence of drug resistance in the context of disease surveillance $(44,45)$. Nowadays, diverse stochastic models are used as tools for estimating probability distributions of potential outcomes from the understanding of environmental and ecological networks involved in the cycles of vector-borne diseases. These geographic models have allowed disseminating critical information concerning public health surveillance for endemic areas (Figure 3).

In kinetoplastid infections, spatial clustering using GIS technology when applied with accurate diagnostic test, analysis of the phylogenetic distribution of Leishmania spp. together with surveillance has been demonstrated to be of great value in predicting areas of risk. Epidemiological analysis of the transmission cycles in Europe, and in recent kala-azar outbreaks in Nepal, Kenya, and Brazil, have helped to precisely define that the priority of intervention is in the vector control with the use of insecticides, in order to block the transmission cycle of disease in potential risk areas, identified to be priority to reduce the spread of infection (46-49). 


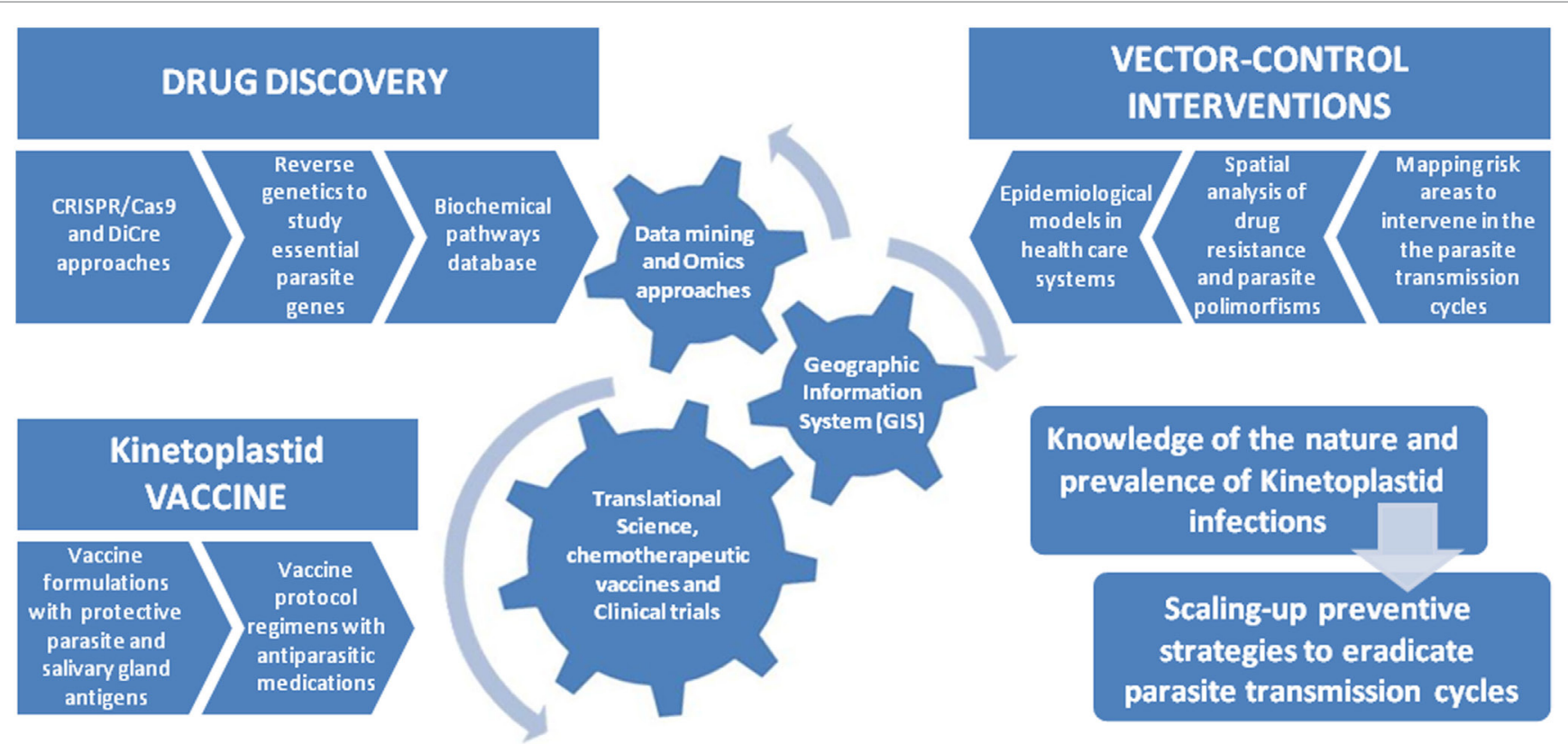

FIGURE 3 | Integrating new approaches into control strategies for kinetoplastid infections. In the past decade, genetic sequencing efforts and manipulation of trypanosomatid genomes have elucidated the characterization of virulence factors and parasite biochemical pathways involved in the pathogenesis disease. These advances allowed the design of new drug targets and therapeutic vaccines capable of reducing the parasitic burden thus controlling the infection and its clinical symptoms. The integration of these tools with epidemiological interventions into public health programs should yield substantial gains in controlling the transmission cycles of these vector-borne diseases. In this line, important studies in the field of vector control interventions have defined a multi-parametric analysis using geographic information system technologies to monitor spatial analysis of drug resistance and parasite polymorphisms to delineate epidemic-prone areas. Those studies have offered a powerful platform for prospecting and development of tools and technologies capable of effectively defining elimination programs in the control and eradication of kinetoplastid diseases.

\section{CONCLUDING REMARKS}

Neglected tropical diseases caused by kinetoplastid protozoan parasites are considered endemic in lower-middle-income economies, although in many countries they are in process of epidemiological containment and elimination. However, the eradication of these diseases is a complex issue that must involve health management policies, coordinated among the affected countries, such as the development of public policies to combat parasitic diseases and improvement of social conditions. This will only be possible through the cooperation of local governments in a participatory search for bilateral relations between public and private interests capable of fostering translational studies that guarantee the access of the population to appropriate treatments. The development of accurate diagnosis capable of identifying these diseases in the acute phase of infection, as well as the identification of parasite polymorphisms and variants of

\section{REFERENCES}

1. Sibley LD. Invasion and intracellular survival by protozoan parasites. Immunol Rev (2011) 240(1):72-91. doi:10.1111/j.1600-065X.2010.00990.x

2. Kling JC, Körner H. Different regulatory mechanisms in protozoan parasitic infections. Int J Parasitol (2013) 43(6):417-25. doi:10.1016/j.ijpara.2013. 02.001

3. Jackson AP, Otto TD, Aslett M, Armstrong SD, Bringaud F, Schlacht A, et al. Kinetoplastid phylogenomics reveals the evolutionary innovations drug resistance, together with application of health care models based on GIS technologies will enable both preventive and therapeutic actions based on new generations of drugs and vaccines currently developed for these neglected diseases.

\section{AUTHOR CONTRIBUTIONS}

AM conceived the manuscript. AAF and AM wrote the manuscript. AAF, KG-P, MPN, KZ, LF, LP, DON, LC, and AM participated in the preparation of the manuscript.

\section{FUNDING}

This work was supported by Conselho Nacional de Desenvolvimento Científico e Tecnológico (CNPQ) and Fundação Carlos Chagas de Amparo à Pesquisa do Estado de Rio de Janeiro (FAPERJ). associated with the origins of parasitism. Curr Biol (2016) 26(2):161-72 doi:10.1016/j.cub.2015.11.055

4. Portman N, Gull K. The paraflagellar rod of kinetoplastid parasites: from structure to components and function. Int J Parasitol (2010) 40(2):135-48. doi:10.1016/j.ijpara.2009.10.005

5. Hotez PJ, Pecoul B, Rijal S, Boehme C, Aksoy S, Malecela M, et al. Eliminating the neglected tropical diseases: translational science and new technologies. PLoS Negl Trop Dis (2016) 10(3):e0003895. doi:10.1371/journal.pntd. 0003895 
6. Majumder HK, de Souza W, Chang KP. Target identification and intervention strategies against kinetoplastid protozoan parasites. Mol Biol Int (2011) 2011:185413. doi:10.4061/2011/185413

7. Hotez PJ. Blue marble health and "the big three diseases": HIV/AIDS, tuberculosis, and malaria. Microbes Infect (2015) 17(8):539-41. doi:10.1016/j. micinf.2015.05.004

8. Pedrique B, Strub-Wourgaft N, Some C, Olliaro P, Trouiller P, Ford N, et al. The drug and vaccine landscape for neglected diseases (2000-11): a systematic assessment. Lancet Glob Health (2013) 1(6):e371-9. doi:10.1016/ S2214-109X(13)70078-0

9. Horstick O, Tozan Y, Wilder-Smith A. Reviewing dengue: still a neglected tropical disease? PLoS Negl Trop Dis (2015) 9(4):e0003632. doi:10.1371/ journal.pntd.0003632

10. Gilbert IH. Drug discovery for neglected diseases: molecular target-based and phenotypic approaches. J Med Chem (2013) 56(20):7719-26. doi:10.1021/ jm $400362 b$

11. Morgan GW, Hall BS, Denny PW, Carrington M, Field MC. The kinetoplastida endocytic apparatus. Part I: a dynamic system for nutrition and evasion of host defences. Trends Parasitol (2002) 18(11):491-6. doi:10.1016/ S1471-4922(02)02391-7

12. Ouaissi A, Ouaissi M. Molecular basis of Trypanosoma cruzi and Leishmania interaction with their host(s): exploitation of immune and defense mechanisms by the parasite leading to persistence and chronicity, features reminiscent of immune system evasion strategies in cancer diseases. Arch Immunol Ther Exp (Warsz) (2005) 53(2):102-14.

13. Fairlamb AH, Gow NA, Matthews KR, Waters AP. Drug resistance in eukaryotic microorganisms. Nat Microbiol (2016) 1(7):16092. doi:10.1038/ nmicrobiol.2016.92

14. Alsford S, Kelly JM, Baker N, Horn D. Genetic dissection of drug resistance in trypanosomes. Parasitology (2013) 140(12):1478-91. doi:10.1017/ S003118201300022X

15. Paucar R, Moreno-Viguri E, Pérez-Silanes S. Challenges in Chagas disease drug discovery: a review. Curr Med Chem (2016) 23(28):3154-70. doi:10.2174/ 0929867323999160625124424

16. Chatelain E. Chagas disease drug discovery: toward a new era. J Biomol Screen (2015) 20(1):22-35. doi:10.1177/1087057114550585

17. Cullen DR, Mocerino M. A brief review of drug discovery research for human African trypanosomiasis. Curr Med Chem (2017) 24(7):701-17. doi:10.2174/0929867324666170120160034

18. Babokhov P, Sanyaolu AO, Oyibo WA, Fagbenro-Beyioku AF, Iriemenam NC. A current analysis of chemotherapy strategies for the treatment of human African trypanosomiasis. Pathog Glob Health (2013) 107(5):242-52. doi:10.1179/2047773213Y.0000000105

19. Kuepfer I, Schmid C, Allan M, Edielu A, Haary EP, Kakembo A, et al. Safety and efficacy of the 10-day melarsoprol schedule for the treatment of second stage Rhodesiense sleeping sickness. PLoS Negl Trop Dis (2012) 6(8):e1695. doi:10.1371/journal.pntd.0001695

20. Schmid C, Kuemmerle A, Blum J, Ghabri S, Kande V, Mutombo W, et al. In-hospital safety in field conditions of nifurtimox eflornithine combination therapy (NECT) for T. b. gambiense sleeping sickness. PLoS Negl Trop Dis (2012) 6(11):e1920. doi:10.1371/journal.pntd.0001920

21. Mesu VKBK, Kalonji WM, Bardonneau C, Mordt OV, Blesson S, Simon F, et al. Oral fexinidazole for late-stage African Trypanosoma brucei gambiense trypanosomiasis: a pivotal multicentre, randomised, non-inferiority trial. Lancet (2018) 391(10116):144-54. doi:10.1016/S0140-6736(17)32758-7

22. Jacobs RT, Nare B, Wring SA, Orr MD, Chen D, Sligar JM, et al. SCYX-7158, an orally-active benzoxaborole for the treatment of stage 2 human African trypanosomiasis. PLoS Negl Trop Dis (2011) 5(6):e1151. doi:10.1371/journal. pntd.0001151

23. Mugnier MR, Stebbins CE, Papavasiliou FN. Masters of disguise: antigenic variation and the VSG coat in Trypanosoma brucei. PLoS Pathog (2016) 12(9):e1005784. doi:10.1371/journal.ppat.1005784

24. Castillo-Acosta VM, Balzarini J, González-Pacanowska D. Surface: a therapeutic opportunity for diseases. Trends Parasitol (2017) 33(10):775-87. doi:10.1016/j.pt.2017.06.009

25. Zaioncz S, Khalil NM, Mainardes RM. Exploring the role of nanoparticles in amphotericin B delivery. Curr Pharm Des (2017) 23(3):509-21. doi:10.2174/ 1381612822666161027103640
26. Jha TK. Drug unresponsiveness \& combination therapy for kala-azar. Indian J Med Res (2006) 123(3):389-98.

27. Sundar S, Chakravarty J. Paromomycin in the treatment of leishmaniasis. Expert Opin Investig Drugs (2008) 17(5):787-94. doi:10.1517/13543784. 17.5.787

28. Andrade HM, Toledo VP, Pinheiro MB, Guimarães TM, Oliveira NC, Castro JA, et al. Evaluation of miltefosine naturally infected with $L$. infantum (=L. chagasi) in Brazil. Vet Parasitol (2011) 181(2-4):83-90. doi:10.1016/j. vetpar.2011.05.009

29. Luque-Ortega JR, Rivas L. Miltefosine (hexadecylphosphocholine) inhibits cytochrome C oxidase in Leishmania donovani promastigotes. Antimicrob Agents Chemother (2007) 51(4):1327-32. doi:10.1128/AAC.01415-06

30. Rakotomanga $\mathrm{M}$, Blanc S, Gaudin K, Chaminade P, Loiseau PM. Miltefosine affects lipid metabolism in Leishmania donovani promastigotes. Antimicrob Agents Chemother (2007) 51(4):1425-30. doi:10.1128/AAC. 01123-06

31. Benaim G, García-Marchán Y, Reyes C, Uzcanga G, Figarella K. Identification of a sphingosine-sensitive $\mathrm{Ca} 2+$ channel in the plasma membrane of Leishmania mexicana. Biochem Biophys Res Commun (2013) 430(3):1091-6. doi:10.1016/j.bbrc.2012.12.033

32. Doyle MA, MacRae JI, De Souza DP, Saunders EC, McConville MJ, Likić VA. LeishCyc: a biochemical pathways database for Leishmania major. BMC Syst Biol (2009) 3:57. doi:10.1186/1752-0509-3-57

33. Biagiotti M, Dominguez S, Yamout N, Zufferey R. Lipidomics and antitrypanosomatid chemotherapy. Clin Transl Med (2017) 6(1):27. doi:10.1186/ s40169-017-0160-7

34. Ritzefeld M, Wright MH, Tate EW. New developments in probing and targeting protein acylation in malaria, leishmaniasis and African sleeping sickness. Parasitology (2017) 8:1-18.

35. Beaumier CM, Gillespie PM, Hotez PJ, Bottazzi ME. New vaccines for neglected parasitic diseases and dengue. Transl Res (2013) 162(3):144-55. doi:10.1016/j.trsl.2013.03.006

36. Duncan SM, Jones NG, Mottram JC. Recent advances in Leishmania reverse genetics: manipulating a manipulative parasite. Mol Biochem Parasitol (2017) 216:30-8. doi:10.1016/j.molbiopara.2017.06.005

37. Raman VS, Duthie MS, Fox CB, Matlashewski G, Reed SG. Adjuvants for Leishmania vaccines: from models to clinical application. Front Immunol (2012) 3:144. doi:10.3389/fimmu.2012.00144

38. Alvar J, Croft SL, Kaye P, Khamesipour A, Sundar S, Reed SG. Case study for a vaccine against leishmaniasis. Vaccine (2013) 31(Suppl 2):B244-9. doi:10.1016/j.vaccine.2012.11.080

39. Cecílio P, Pérez-Cabezas B, Fernández L, Moreno J, Carrillo E, Requena JM, et al. Pre-clinical antigenicity studies of an innovative multivalent vaccine for human visceral leishmaniasis. PLoS Negl Trop Dis (2017) 11(11):e0005951. doi:10.1371/journal.pntd.0005951

40. Dumonteil E, Bottazzi ME, Zhan B, Heffernan MJ, Jones K, Valenzuela JG, et al. Accelerating the development of a therapeutic vaccine for human Chagas disease: rationale and prospects. Expert Rev Vaccines (2012) 11(9): 1043-55. doi:10.1586/erv.12.85

41. Seid CA, Jones KM, Pollet J, Keegan B, Hudspeth E, Hammond M, et al. Cysteine mutagenesis improves the production without abrogating antigenicity of a recombinant protein vaccine candidate for human Chagas disease. Hum Vaccin Immunother (2017) 13(3):621-33. doi:10.1080/21645515.2016. 1242540

42. Konduri V, Halpert MM, Liang D, Levitt JM, Cruz-Chan JV, Zhan B, et al. Genetic adjuvantation of a cell-based therapeutic vaccine for amelioration of chagasic cardiomyopathy. Infect Immun (2017) 85(9):e127-117. doi:10.1128/ IAI.00127-17

43. DebRoy S, Prosper O, Mishoe A, Mubayi A. Challenges in modeling complexity of neglected tropical diseases: a review of dynamics of visceral leishmaniasis in resource limited settings. Emerg Themes Epidemiol (2017) 14:10. doi:10.1186/s12982-017-0065-3

44. Bergquist NR. Vector-borne parasitic diseases: new trends in data collection and risk assessment. Acta Trop (2001) 79(1):13-20. doi:10.1016/ S0001-706X(01)00099-7

45. Palaniyandi M. The role of remote sensing and GIS for spatial prediction of vector-borne diseases transmission: a systematic review. J Vector Borne Dis (2012) 49(4):197-204. 
46. Ryan JR, Mbui J, Rashid JR, Wasunna MK, Kirigi G, Magiri C, et al. Spatial clustering and epidemiological aspects of visceral leishmaniasis in two endemic villages, Baringo District, Kenya. Am J Trop Med Hyg (2006) 74(2): 308-17.

47. Khanal B, Picado A, Bhattarai NR, Van Der Auwera G, Das ML, Ostyn B, et al. Spatial analysis of Leishmania donovani exposure in humans and domestic animals in a recent kala azar focus in Nepal. Parasitology (2010) 137(11):1597-603. doi:10.1017/S0031182010000521

48. Tsokana CN, Sokos C, Giannakopoulos A, Mamuris Z, Birtsas P, Papaspyropoulos K, et al. First evidence of Leishmania infection in European brown hare (Lepus europaeus) in Greece: GIS analysis and phylogenetic position within the Leishmania spp. Parasitol Res (2016) 115(1):313-21. doi:10.1007/s00436-015-4749-8

49. Sevá AD, Mao L, Galvis-Ovallos F, Tucker Lima JM, Valle D. Risk analysis and prediction of visceral leishmaniasis dispersion in São Paulo State, Brazil.
PLoS Negl Trop Dis (2017) 11(2):e0005353. doi:10.1371/journal.pntd. 0005353

Conflict of Interest Statement: The authors declare that the research was conducted in the absence of any commercial or financial relationships that could be construed as a potential conflict of interest.

Copyright @ 2018 Filardy, Guimarães-Pinto, Nunes, Zukeram, Fliess, Pereira, Oliveira Nascimento, Conde and Morrot. This is an open-access article distributed under the terms of the Creative Commons Attribution License (CC BY). The use, distribution or reproduction in other forums is permitted, provided the original author(s) and the copyright owner are credited and that the original publication in this journal is cited, in accordance with accepted academic practice. No use, distribution or reproduction is permitted which does not comply with these terms 